\title{
Visualizing expertise: Collaborative Pathway Modeling as a methodology for conveying community-driven strategies for change
}

\section{FoodDignity}

\author{
Monica Hargraves a * \\ Cornell University \\ Cecilia D enning $b$ \\ Action Resources International
}

Submitted September 20, 2017 / Revised D ecember 5, 2017, and February 23, 2018 /

Accepted February 24, 2018 / Published online July 18, 2018

Citation: Hargraves, M., \& D enning, C (2018). Visualizing expertise: Collaborative Pathway Modeling as a methodology for conveying community-driven strategies for change. Journal of A grialture, Food Systems, and Community D evelopment, 8(Suppl. 1), 101-115. https:/ / doi.org/ 10.5304/ jafscd.2018.08A.005

Copyright @ 2018 by the Authors. Published by the Lyson Center for Civic Agriculture and Food Systems. Open access under CC BY license.

\begin{abstract}
The Food Dignity project brought teams from five community-led organizations working on local food systems together with researchers from four academic institutions, to learn from community strategies for building sustainable local food systems and improving food justice. This reflective
\end{abstract}

a* Corresponding author: Monica Hargraves, Associate Director for Evaluation Partnerships, Cornell O ffice for Research on Evaluation, Cornell University; 35 Thornwood D rive, Suite 200, Room 150-C; Ithaca, New York 14850 USA; mjh51@ cornell.edu

b Cecilia D enning, Evaluation and Special Projects, Action Resources International; P.O. Box 536; Laramie, Wyoming 82073 USA; cecilia@ actionresources.ngo

\section{Disclosures}

Hargraves and D enning serve on the board of directors of and have consulted to Action Resources International, the sponsoring organization for Feeding Laramie Valley, one of the community organizations partnering in the Food Dignity proiect. essay describes the emergence and refinement, within this context, of a values-driven methodology for surfacing, protecting, and conveying the strategic thinking and theories of change held by community practitioners. Knowledge utilization is too often viewed as a one-way street in which researchderived knowledge is expected to infuse and improve practice, without sufficient focus and mechanisms to ensure that practice-derived knowledge is

\section{Contributors and Supporting Agencies}

Blue Mountain Associates; Feeding Laramie Valley; Whole Community Project; East New Y ork Farms!; D ig D eep Farms; University of Wyoming; Cornell University; U.S. D epartment of Agriculture National Institute of Food and Agriculture (USD A NIFA).

\section{Funding Disclosure}

Food Dignity (http:/ / www.fooddignity.org) was supported by Agriculture and Food Research Initiative Competitive Grant no. 2011-68004-30074 from the USD A National Institute of Food and Agriculture. 
valued and brought forward. Collaborative Pathway Modeling (CPM) addresses this gap by offering a practical tool for capturing and presenting practitioners' theories of change. Importantly, the models that are produced are not just useful as tools for research. They have been valuable and useful to the community organizations themselves, underscoring a central commitment in CPM to equity and respect for community expertise and intellectual property. In this paper we describe the origins and development of CPM and its researchderived approach to program modeling, situate CPM relative to calls for greater community involvement in research, and present the values and process that define the methodology. We share stories from developing the community partner models, and conclude with reflections on the nature of the work and its larger potential for bringing forward essential diverse sources of knowledge in many arenas.

\section{Keywords}

Collaborative Pathway Modeling; Collaborative Research; Community Knowledge; Practitioner Expertise; Theory of Change; Program Modeling; Community-based Participatory Research; Food Dignity

\section{Introduction}

Efforts to improve the strength, equity, and sustainability of community food systems-as with efforts to address many contemporary community problems - face a complex mix of systemic and local challenges. It stands to reason that relevant, effective solutions would require the expertise of community leaders and others with lived experience and knowledge of their community realities, history, culture, obstacles, strengths, and priorities. Researchers and funders in many fields have come to recognize the value of community expertise, as reflected in widespread calls for new or stronger practices such as community-academic partnerships, community-based participatory research (CBPR), and community involvement in research and implementation science (Ahmed \& Palermo, 2010; D rahota et al., 2016; Green, 2001; G reen \& Mercer, 2001; Leviton \& Trujillo, 2016; Lobb \& Colditz, 2013; Pine \& de Souza, 2013; Wallerstein
\& D uran, 2010; Wandersman, Alia, Cook, Hsu, \& Ramaswamy, 2016). The value that is added by participatory research is summarized well by Minkler (2005), who identifies numerous ways that the quality, relevance, and validity of research can be improved through CBPR.

It is also important to recognize - as some of the above scholars do, explicitly - that community and practitioner experts originate solutions of their own. That is, it is not just that community voices need to be included in research projects in order to generate research that is more relevant, viable, and effective in addressing community problems, but that experienced community practitioners have answers and ideas- program designs and policy recommendations - of their own, drawing on their distinct expertise and knowledge. Indeed, the Food Dignity action research project was based on the recognition that practitioners are implementing important solutions that researchers can learn from. In practice, however, community-generated programs face numerous challenges. As Tseng claims, the "past 15 years have not created a meaningful role for practitioners in building evidence agendas. Instead, evidence agendas have been largely under the province of policymakers and researchers" (Tseng, 2015, "Where are we going," item 4). Wallerstein and Duran list "the privileging of academic knowledge" as one of the six core challenges of translational research (Wallerstein \& D uran, 2010, p. S41). Chen and Turner (2012) claim that formal theory from academia is favored over stakeholder theories, and that practitionerdeveloped programs have been systematically discounted compared to formal theory-based interventions- less likely to be studied, funded, or included in the published literature - although they tend to be favored by practitioners and community stakeholders as more relevant and practical. The CPM process described in this essay offers a practical tool for bringing practitioner theories of change forward, an important step toward obtaining due consideration for funding, evaluation, and evidence-development for practitioner-derived solutions.

CPM is a structured, values-driven process for surfacing and articulating the insights, knowledge, and expertise of groups and individuals designing 
community-driven solutions to community problems. The strength of CPM is that it combines two things: an ethics-driven approach that elevates and protects the expertise derived from lived experience, and a structured process that yields a program model and visual theory of change. The models that emerge are well suited to evaluation, research-practice integration, and related endeavors that benefit from an articulated framework of organizing concepts (Trochim et al., 2016; Urban \& Trochim, 2009).

An important point is that both the process and results of CPM are also valuable to the community leaders and community members whose expertise is brought forward. The intricate models that emerged from the CPM process with Food Dignity community partners have facilitated internal and external stakeholder communication and understanding, strengthened grant-writing and proposal development, and provided a valued bridge between the complex expertise of community leaders and external stakeholders.

In this reflective essay, we explain and describe how we developed CPM in the context of the Food Dignity action research project, and how and why it has been valuable to both researchers and practitioners. We begin, in the following section, with the origins of CPM, with dual roots in the research-tested pathway modeling methods central to the Systems Evaluation Protocol, and insights from community partners in the Food Dignity action research project that shaped the CPM process. Then we describe the development of CPM and how the process evolved as we extended the effort beyond the initial projects, explaining significant challenges using examples from the Food Dignity work. This narrative approach is complemented by a step-by-step summary of the CPM process in the Appendix to underscore and clarify the process. The values foundation for CPM guides the way the work is done, and is discussed next. We conclude with reflections on the CPM experience with community partners and recommendations for future work.

\section{Origins of Collaborative Pathway Modeling}

CPM utilizes the program modeling process that is central to the evaluation planning approach developed by the Cornell O ffice for Research on Evaluation (CORE), formalized as the Systems Evaluation Protocol (Trochim et al., 2016). The protocol and companion software system called the Netway were developed with research grants from the National Science Foundation (NSF Awards \#0535492 and \#0814364) and were tested and refined in multiple cohorts of evaluation partnerships with various U.S. education and outreach programs.

The reasoning behind the particular kind of program modeling used in the protocol comes from the recognition that before planning a program evaluation, it is essential to establish a clear and detailed understanding of what that program is and how it works: what program participants do or experience as part of the program, what kinds of early changes these activities lead to and what changes unfold later, and how the activities in the program work to bring about those particular changes and set off the whole process that ultimately leads to expected impacts. Outsiders to a program may not always have that level of understanding of a program. A program model is one way that that information and insight can be communicated. Many evaluation strategies involve developing a logic model, in which lists of program inputs, activities, and short-, mid-, and long-term outcomes (the anticipated changes) are laid out in columns. Logic models are used in many evaluations, are often required as part of grant proposals, and in general are useful as a way of providing concise program information in a standard format. Logic models provide a great deal of information, but are not able to present the reasoning about how change works- that is, how and why particular activities are believed to contribute to or cause the changes listed in the outcome columns. Those causal connections - which make up the "theory of change" for a program - explain how a program is believed to work and provide a foundation for devising an evaluation to investigate how well it is working, how to make it work better, or how to make it work in different circumstances.

The Systems Evaluation Protocol uses pathway modeling, which is a visual type of program logic modeling that highlights the underlying theory of change and presents it in specific detail. The theory 
of change spells out how the activities of a program are expected to lead to its larger, long-term goals. In a pathway model, program activities and outcomes appear as boxes with concise descriptive text, and arrows connect each program activity to the short-term outcome(s) to which it contributes. Additional arrows link each outcome to the other outcome(s) that it contributes to, tracing the pathways of change and convergence and the incremental manifestations of progress implied by the theory of change underlying the program design. The resulting diagram contains a wealth of information about how change is believed to unfold over the course of a program and beyond, and about the specific outcomes expected to emerge in the process (see Figure 1 for an example).

As Figure 1 shows, these diagrams can be very complicated, and may be daunting at first glance. Complex pathway models, however, reflect the complexity of the work itself. The challenge in pathway modeling is to make the model detailed enough to convey specific insights and components of change, while not making the model so dense that the details become unreadable. It takes time to absorb the information they contain. (The small image size makes the model in Figure 1 difficult to read, but our intent is to provide a quick view of what a pathway model can look like. A larger version of the model in Figure 1 can be viewed at https:/ / www.fooddignity.org/ collaborative-pathway-models.)

Pathway models in the Systems Evaluation Protocol form the foundation for evaluation decision-making by laying out the essential elements of the program design, theory of change,

Figure 1. Collaborative Pathway Model Poster of the Whole Community Project (Ithaca, New York)

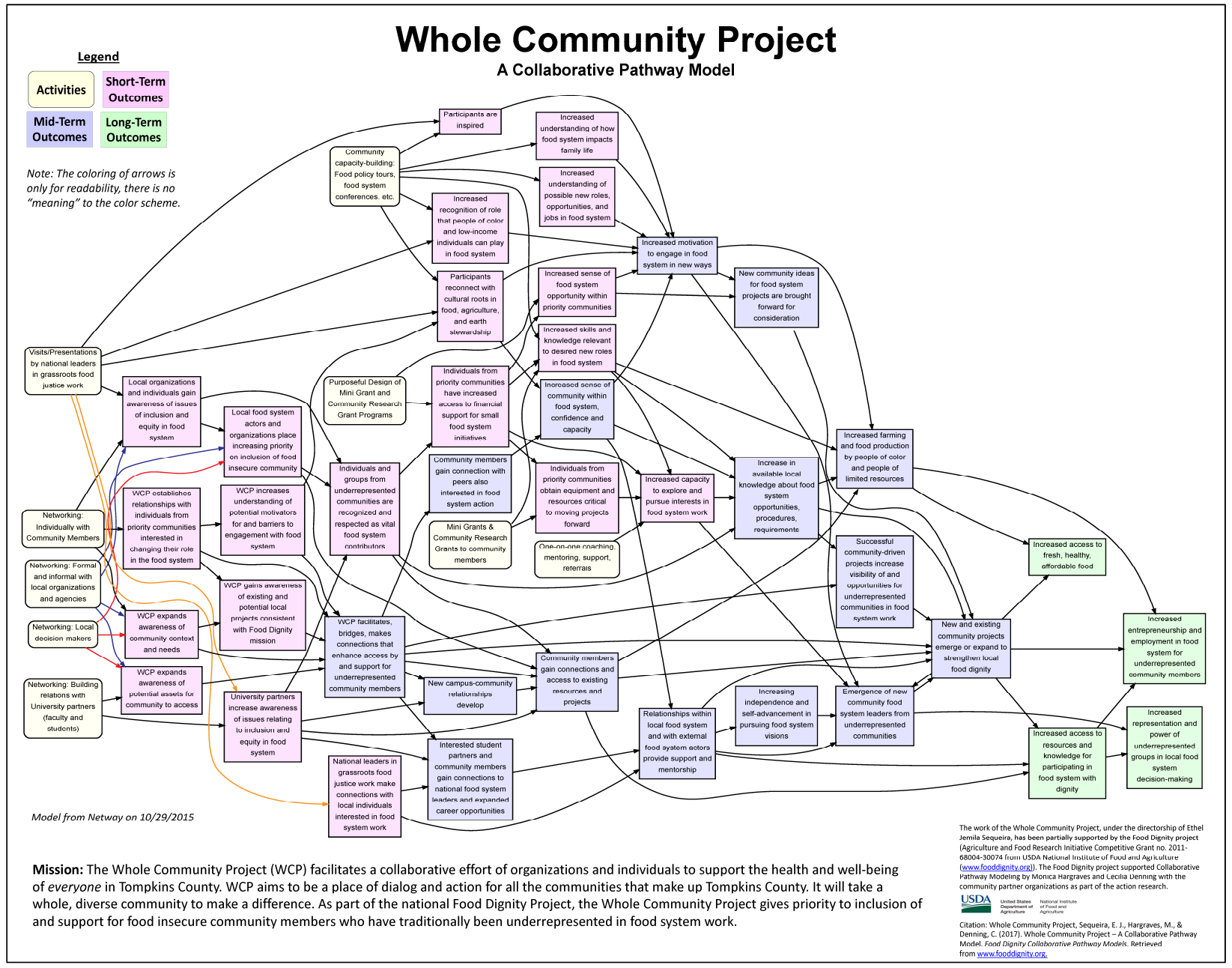


and activities and outcomes (Trochim et al., 2016). An evaluation plan then focuses on selected parts of the program process, guiding the collection of data that will be most useful for program staff, funders, and other stakeholders. Pathway models can also be valuable for integrating research and practice by providing a framework in which existing research evidence can be mapped onto a program model and aligned with locally generated evidence from the program evaluation to provide a more complete evidence base for a program than is typically available from local program evaluations (Urban \& Trochim, 2009). As a staff member of CORE, as well as part of the Food Dignity research team, Hargraves is familiar with pathway modeling, making it a natural choice for the Food Dignity project.

As the work proceeded, the model development approach was refined to serve a new and important purpose, bringing forward the expertise and theories of community practitioners. It is this purpose that marks the distinction between pathway modeling for purposes of evaluation and program development, as in the Systems Evaluation Protocol, and pathway modeling specifically aimed at elevating practitioner expertise and theories of change, which we called Collaborative Pathway Modeling. The technical structure of pathway modeling utilized in the Systems Evaluation Protocol is retained in CPM, but the process of gathering information and building the model in a particular kind of partnership with community program leaders is the distinguishing feature of $\mathrm{CPM}$. The process combines the experience and expertise of the pathway modeling team and the designers and leaders of the community-based program, jointly creating a technically strong program model that serves the specific purpose of articulating the practitioners' theories of change. In this way CPM is designed to help counterbalance the prevailing system, which favors researcher expertise above others. Including "collaborative" in its name connects with collaborative and culturally responsive evaluation approaches, which have been described as "acknowledg[ing] the importance of valuing stakeholder knowledge as part of a larger effort to better understand a program's operation and impact" (Askew, Beverly, \& Jay, 2012, p. 552).
This purpose of CPM aligned well with the Food Dignity project, a five-year (2011-2016) action research project funded by the U.S. D epartment of Agriculture's National Institute of Food and Agriculture (USD A NIFA) to explore community-led approaches to food justice and sustainable local food systems. It was predicated on the need to learn from grassroots community organizations designing local approaches to food system challenges and inequities. "Food dignity as a premise and Food Dignity as a research project are both steeped in recognizing that community people hold the knowledge and ability to ask the right questions and find the right answers to their own needs" (Porter, Herrera, Marshall, \& Woodsum, 2014, p. 124).

Five U.S. community organizations joined the Food Dignity project as action research partners: Blue Mountain Associates, on the Wind River Indian Reservation in Wyoming; Dig D eep Farms, in Alameda County, California; East New Y ork Farms!, in Brooklyn, New Y ork; Feeding Laramie Valley, in Laramie, Wyoming; and the Whole Community Project, in Ithaca, New York (see http:/ / www.fooddignity.org for more information on these partner organizations). Their common thread was dedication to strengthening sustainable local food systems and to food justice. Each organization and its programs is unique, however, reflecting the characteristics and priorities of their communities, as well as their organizational strengths and priorities.

The research challenge in the Food Dignity project involved how to surface and articulate the expertise and strategies driving these communitydriven approaches. Extensive qualitative data, including interviews, digital storytelling, case studies, photo and video documentary evidence, and quantitative data on garden harvests and other elements made up the bulk of the planned research. Pathway modeling, and more specifically $\mathrm{CPM}$, provided not just an additional unique form of data about the strategies in use in the five community programs, but a community-driven framework for organizing and analyzing other project data by identifying themes that were part of the community organization's work. 


\section{Emergence and Development of Collaborative Pathway Modeling}

The first effort to use pathway modeling in Food Dignity took place in early 2014 with the Whole Community Project (WCP), directed by Jemila Sequeira. At that time there was no comprehensive plan for pathway modeling in Food Dignity; it was seen simply as a way to pull together a structured representation of the complex and responsive work of WCP for communication and reporting purposes. The WCP was based in Ithaca, New Y ork, which is also home to Cornell University and CORE, where Hargraves is based. Their proximity made it easy for Sequeira and Hargraves to meet in person, and the model development proceeded as a collaboration between them.

O ut of the rich story-telling and documentation provided by Sequeira, the underlying logic of her hands-on, relationship-focused approach to community organizing began to emerge. Hargraves and Sequeira were able to work through multiple iterations of the model over time, eventually arriving at a model that met with Sequeira's approval-and that one of her colleagues commented looked like "the inside of Jemila's brain!" (J. Sequeira, personal communication). The critical contribution of Sequeira's extensive and strategic networking with community members, local and national organizations, and academics is particularly visible in the model (see Figure 1 or the larger WCP model at http:/ / fooddignity.org/ collabora tive-pathway-models) and helped convey the roles and relevance of an array of informal meetings, conversations, and related activities that had previously been discounted or unrecognized.

On the strength of the productivity of the modeling effort and the value- to Sequeira, the WCP, and the Food Dignity project- of the visually represented theory of change that emerged from that work, the Food Dignity leadership team proposed in October 2014 to extend the effort to all five community partner organizations. G ayle Woodsum, a community organizer with more than three decades of experience in grassroots organizing who was serving as community liaison for the Food D ignity project, had been interested in trying pathway modeling herself, based on its success with WCP. She pointed out the powerful potential of this kind of pathway modeling for articulating expertise in community-based work that so often goes unheard and undervalued. It was this insight that launched the CPM work in Food Dignity and anchored the process in its value foundation and purpose.

Cecilia D enning joined Hargraves in late 2014 to strengthen and support the newly planned work with community partners, forming a two-person CPM team. Given the distances between the community partner organizations, models for the remaining partner programs were developed through varying combinations of in-person visits and follow-up phone meetings. The model for Feeding Laramie Valley (FLV), directed by G ayle Woodsum, was the second to be developed, drawing on an initial in-person meeting in 0 ctober 2014 and evolving through several rounds of revisions over numerous phone meetings with Hargraves, D enning, and Woodsum until it met with Woodsum's approval.

The experiences of developing models for Sequeira's and Woodsum's organizations laid the groundwork for extending the project to the other three community organizations, and we moved to establish a more standardized procedure for the collaborative process thereafter. For the remaining three community partners we had the time and funds for one two-day site visit for in-person work for each organization, so we worked to make that opportunity as productive as possible by reviewing written reports and website materials in advance to identify potential activities and outcomes for the anticipated model. This pre-analysis was sketched into a temporary prototype model, essentially a skeleton to speed the process of creating a full model during the site visit. The in-person meetings amounted to semistructured group interviews, soliciting stories and explanations of what the staff and leadership do, why it matters, what differences their activities make and why, how change takes place, what barriers exist, and whatever else they felt was relevant to understand how and why their programs work the way they do. (For ease of reference, the steps of CPM are summarized in a table in the Appendix.)

Throughout these discussions and iterations, the most important responsibility for us, the CPM 
team, was to listen deeply. We were outsiders to the communities and programs we were modeling, and it was incumbent on us to represent what they perceived to be true, not what we thought made sense or could distill from the information they gave us. This marks a subtle but important distinction between the goals of CPM and Classical Grounded Theory, to which it might otherwise seem similar. In Classical G rounded Theory, researchers collect extensive information from stakeholders that is then analyzed, coded, and refined in a systematic, iterative process in order for the researchers to develop a theory of the phenomenon under study (Evans, 2013; Glaser \& Strauss, 1965). By contrast, the goal of CPM is to bring forward the practitioners' theory of change for a program they have designed or are involved in delivering. This demanded from us a particular kind of critical listening. On the one hand, we had to question and scrutinize what we were hearing in order to ensure that the logical connections we were building were complete so that the model would be technically strong. On the other hand, we had to listen as openly as we could for things that necessarily were foreign to us, in order to detect the crucial mechanisms and characteristics of the change process. The process of listening deeply, asking questions, and revising and checking in with the contributors repeatedly until we had it right in their eyes was essential (see Figure 2).

It was a priority for each site visit to develop an initial full model in time for the contributors' inperson review before the end of the visit. Presenting the model to the contributors in person gave us a chance for richer discussions, ensuring that they were familiar with how pathway models work in general so that their feedback on their own pathway model could be precise and well directed. After the site visit, we made revisions that they had suggested and had follow-up phone meetings in order to refine the models further, until the community organizer and primary contributors felt the model offered a good representation of their work, their strategies, and their view of how change unfolds. That commitment- to listening, revising however much was required and even scrapping early versions, and deferring to the judgment of the community organizer- was essential for the quality of the model, not only for ensuring the accuracy of the end product, but also for the authenticity of the process itself. There had to be a foundation of trust in order for information to be shared and stories to be told.

An important feature of the final models is that they are recognized as the intellectual property of the community organizer directing the work and guiding the modeling process, and are the property of the community organization. In each finalized CPM poster 
authorship is specified and is shared between the organization, the community organizer, and the CPM team. Table 1 lists the community organizer and primary contributors to the CPM for each partner organization. The five pathway model posters themselves can be viewed at http:/ / fooddignity.org/ collaborative-pathwaymodels.

\section{What It Takes to Build a Pathway Model: Important Considerations Along the Way} The pathway model diagrams were constructed using the Netway, a free web-based software program developed by CORE to support evaluation planning using the Systems Evaluation Protocol (https:/ / www.evaluationnetway.com). The pathway modeling feature of the Netway makes it easy, from a technical production point of view, to create and revise models. The process of building a collaborative pathway model, or a pathway model in general guided by the Systems Evaluation Protocol, involves attending to a number of features: program scope appropriate for the modeling exercise; individual elements of Activities, Outcomes, and Links indicating direction of contribution or causality; wording for the Activity and O utcome text; and Program Context. The process of developing each of these is described below, with examples from the Food D ignity partner models.

\section{Program Scope}

A critical step in the modeling process is to determine what scope of work will be included in the model. The choice is driven by the vision and communication needs of the program, as there can be both broad and narrow perspectives on the program which may be appropriate for different circumstances. The general goal is that the scope should be large enough to include aspects of the program that are of interest and that are needed to have a full understanding of how the program works, and yet be small enough to allow a level of detail that is informative while still being readable. In the terms used in the Systems Evaluation Protocol, this is a program "boundary" question (Trochim et al., 2016).

The issue of program scope came to the fore most vividly in the modeling of the D ig D eep Farms (DDF) program in California. Because our pre-visit materials were drawn from D DF annual reports and web pages, our skeleton pre-model focused on their farming, food distribution, and workforce-development activities and outcomes. However, the DDF leadership reaction upon seeing the pre-visit sketch was strongly negative. The intense discussions that followed made it clear that D DF is a part of a much larger community change initiative, and- most important- that it is not possible to understand the D DF enterprise unless it is viewed from this larger perspective. We restructured the model extensively in order to present D ig D eep Farms in its larger context; in fact, this became the title of the CPM poster. The result was a larger and more complex model that presented not only the work of DDF itself but its role as a significant pilot and demonstration project for an entirely new way of seeing and addressing complex community challenges involving poverty, lack of access to healthy food, lack of jobs (especially for those reentering the community from prison), and lack of community infrastructure, gathering places, and opportunities. The resulting revised model, while still in need of fine-tuning, met with approval. In

Table 1. Food Dignity Community Partner Organizations and Collaborative Pathway Modeling (CPM) Contributors

\begin{tabular}{ll}
\hline Community Organization and Location & Community Organizer and Primary CPM Contributor \\
\hline Blue Mountain Associates, Wind River Indian Reservation, Wyoming & Dr. Virginia Sutter, James Sutter \\
\hline Dig Deep Farms, Ashland/ Cherryland, California & Capt. Marty Neideffer, Hilary Bass \\
\hline East New York Farms!, Brooklyn, New York & David Vigil, Daryl Marshall \\
\hline Feeding Laramie Valley, Laramie, Wyoming & Gayle Woodsum \\
\hline Whole Community Project, Ithaca, New York & Jemila Sequeira \\
\hline
\end{tabular}


the words of project director Marty Neideffer, responding to the first viewing of the revised CPM developed with his team, "No one has ever gotten us before" (M. Neideffer, personal communication, June 12, 2015).

A ctivities, 0 utcomes, and L inks In principle, a pathway model can be built from left to right (activities through to long-term outcomes), from right to left (working from long-term outcomes back to the activities that launch the work), or from some mix of the two. In practice, in the CPM work of the Food Dignity project it was generally a matter of listening in order to identify activities and the significant big-picture outcomes, both of which tended to be easier to hear, and then filling in the linkages and incremental outcomes in between by listening to stories of change in order to capture the causal story lines, and by asking questions about what difference something made or why it mattered.

The information came together in different ways, depending on the speakers and how they tend to think and view their work. In the case of Blue Mountain Associates, for example, the logic of their work emerged very clearly through Dr. Sutter's explanations of the origins and intentions behind the various parts of their work and how changes unfolded, thus providing natural left-toright accounts for the modeling. In the case of the Whole Community Project, modeling began with Sequeira's clear initial announcement of four key long-term goals toward which all the effort was directed, and then the incremental change process was filled in from many stories about individuals reached by the work, networking efforts by the community organizer at many levels in the community, and so on. The staff and leadership contributing to the East New York Farms! pathway model individually represented several different aspects of their work in the community, and the ways that they described their work focused on the activities composing these organizational aspects. The challenge for modeling was to listen for how these distinct components interacted and reinforced each other so as to yield larger, integrated change.
L anguage and C oncepts

A great deal of detail is necessarily excluded when reducing richly detailed stories and nuanced strategies to a two-dimensional diagram with small boxes of text and connecting arrows. Trying to be as true to the work as possible and faithful to the expertise driving the program design requires considerable care about wording in the space-constrained activity and outcome boxes in the model. Using the language of the community organizers and program staff would be ideal, but this is not always possible. The art of the modeling process is to distill the essencethe underlying crucial concept- and word the text accordingly.

One example of such distillation is from the Feeding Laramie Valley modeling. A central goal of its work is to shift the community paradigm from a top-down charity model for responding to food insecurity to a more mutual enterprise in which the spirit and practice of sharing provides the foundation for improving community food security. Its core program activities are called "Shares" programs: in "Yard Shares," interested community members with yards are supported in developing gardens, with some of the produce shared with food-insecure households; in "FLV Shares," vendors and patrons at the farmers market are invited to contribute fresh quality produce that is given to families lacking fresh, healthy food. In describing these programs, Gayle Woodsum recounted a story from the weekly food-sharing delivery to a Laramie senior center. Several recipients of the weekly food baskets came not just to pick up their fresh produce, but also to contribute other food of their own that they had in abundance- sharing back to the community. The story resonated strongly with $\mathrm{G}$ ayle, and it was clear that it needed to be depicted in the model. As shown in Figure 3, an excerpt from the FLV model, "Softening lines between giver and receiver" is a short-term outcome emerging in the course of its work. Numerous arrows lead into and out of that element in the model, presenting it as a key shift along the way to larger success in changing the overall community approach to improving food security. 
Program C ontext

The finishing touch on the final CPM

posters was the

addition of text

presenting informa-

tion about each

program's context. In

the form of brief

paragraphs or bullet

points, this informa-

tion provides addi-

tional information

that a viewer needs to

know to understand

important features

shaping program

design. In the case of

the Food Dignity

partners, this infor-

mation was either

provided by them

directly or was drawn

from existing written

materials and

approved by them.

\section{Guiding Values}

One of the defining features of CPM is the set of values that shape the process. The foundational premise of CPM is that first-person, lived experience yields a unique and valuable type of expertise. The goal and commitment of CPM are to bring that expertise to the surface, translate it accurately and faithfully, and protect the intellectual property rights of those who hold that expertise.

Trust is essential to this process but is not automatically achieved. Why should community organizers trust and share with outside researchers their hard-won expertise, with the all-too-common history of seeing it misused, or scooped up and taken away for analysis and publication while they continue to battle the challenges they are working to solve? (Cochran et al., 2008; Corbie-Smith, Thomas, \& St. G eorge, 2002; Christopher, Watts, McCormick, \& Young, 2008; Porter et al., 2014). The effort to establish equitable, collaborative Valley Model
Figure 3. Excerpt Showing a Key Short-Term Outcome in the Feeding Laramie

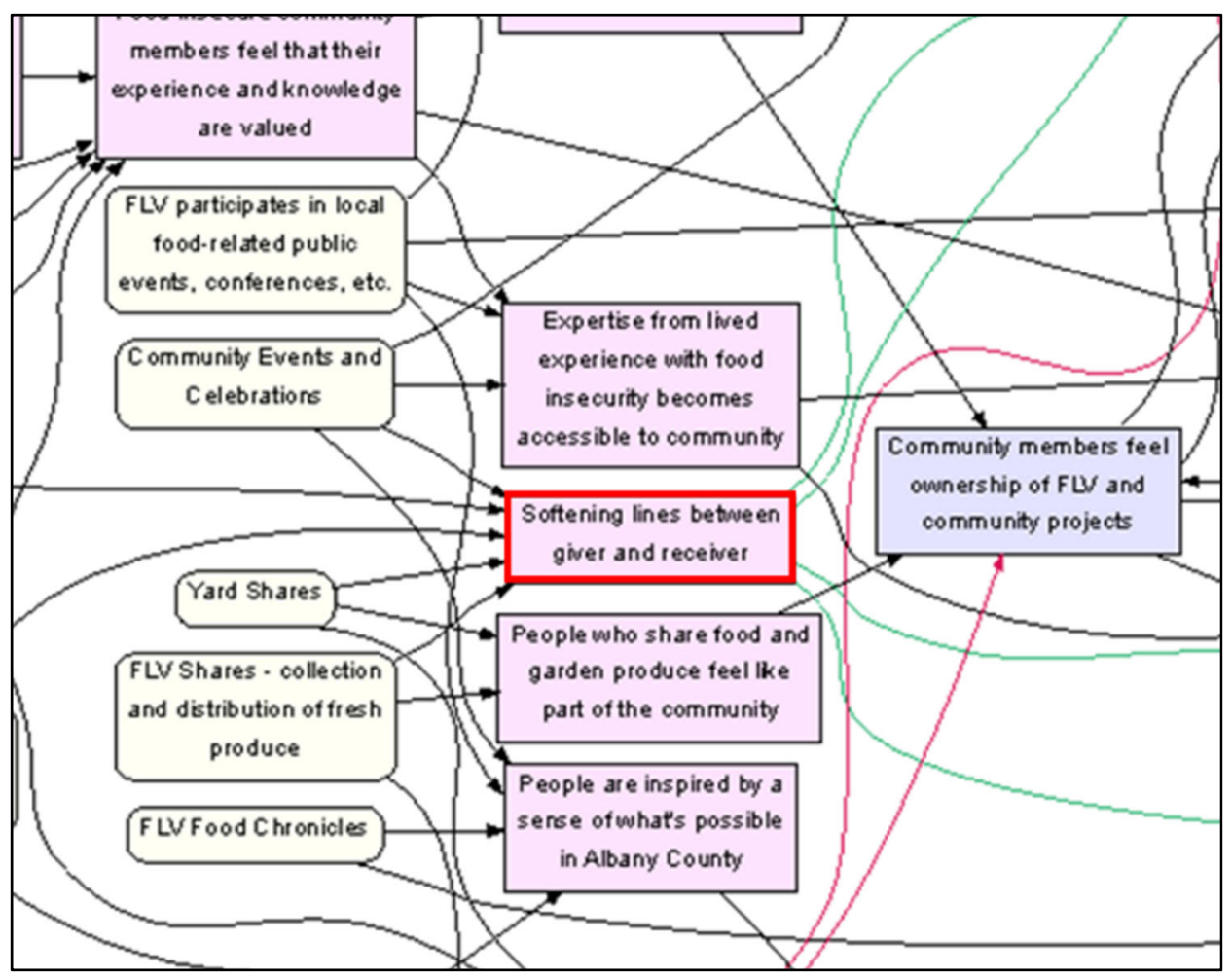

relationships and trust among the community and academic partners in the Food Dignity project was an active commitment and challenge in the early years of the project, requiring ongoing recommitment. Nevertheless, in 2014 Food Dignity was awarded the Community-Campus Partnerships for Health annual award, which included praise for the "partners' honesty and selfreflection in describing their challenges and how they dealt with them" (Community-Campus Partnerships for Health, n.d., para. 1).

These Food Dignity project efforts contributed an important basis for the CPM work as it emerged in the final two years of work. Building on that foundation, a critical component of the CPM effort was clear communication about its underlying specific values. These values, presented in Table 2, served as guiding principles shaping the way the CPM team prepared for and worked with community organizers to come to understand the strategies underlying the community programs, and to build and revise models to represent the programs accurately. Although we had not enumerated them 
when we began the CPM work, they were present from the beginning because they were integral to the goals of the modeling work - to bring to the surface, highlight, and protect the insights and strategies of the community organizers. As we worked through the series of modeling collaborations, the importance of these underlying principles became more and more clear, and we were able to summarize them succinctly.

\section{Reflections and Recommendations}

While the commitments and associated operating principles underlying CPM look tidy when laid out in Table 2, doing the actual work can feel difficult and messy. Particularly under the very tight turnaround time necessitated by the two-day site visits, the thinking and model-crafting work can be very challenging. From the myriad stories, examples, vignettes, and explanations that we heard over the course of our in-person interviews, we worked to distill, synthesize, and integrate themes while still maintaining the distinctness of ideas and outcomes we had heard mentioned. It was invaluable with this turnaround schedule that the CPM team had technical facility and extensive experience with causal pathway modeling. The quality of the models was also strengthened by both of us listening closely so that we could compare notes and interpretations after the interviews. From this material we highlighted recurring themes and individual elements and then laid out elements and connective threads. Going from individual elements and story lines to an articulated model is a big step, however, and it felt almost sculptural-adding, carving away, linking, polishing - until the model cohered and began to do justice to the knowledge that had been shared with us.

Aside from the intensity of the listening and envisioning process, an important challenge we wrestled with was the issue of validity. No matter how carefully we listened and asked questions, it is unreasonable to expect that, if another team of CPM model-builders were to conduct the same two-day site visit and talk to the same people, they would develop the same model exactly in every detail that we had. There is a different kind of validity that this process seeks. Rather than exact, replicable precision of every individual detail, we sought accuracy of representation in the eyes of those whose ideas and causal theories we were trying to illustrate. It was critical to have thoughtful engagement and review from our community partners. This amounted to a type of expert validity, and this was the standard we worked toward.

With thoughtful review by community partners, the end result of the explorations, conversations, questions, distillation, synthesis, and iterative revisions of the CPM effort is a representation of the work and theory of change driving the community program that accurately reflects the community organizer's vision and understanding. From a Food Dignity research project point of view, the models have added a valuable way of understanding and presenting the strategies and expertise of the community partners whose work is the heart of the Food Dignity learning process. The distillation of information in the models lends itself to crossprogram analyses that will be pursued in future work.

Importantly, the end results have been highly valued by the community partners for their own needs. Even just the initial, superficial impressions provided by the models have been validating for organization leaders and staff, as the complexity of each diagram feels like a fitting reflection of the

Table 2. Values and Operating Principles of Collaborative Pathway Modeling

\begin{tabular}{ll}
\hline \multicolumn{1}{c}{ Values } & \multicolumn{1}{c}{ Operating Principles } \\
$\begin{array}{ll}\text { Commitment to presenting the expertise and insights } \\
\text { held by the people doing the work }\end{array}$ & Careful preparation in advance, deep listening \\
$\begin{array}{ll}\text { Commitment to integrity in translation and } \\
\text { communication }\end{array}$ & $\begin{array}{l}\text { Upholding technical standards, logical discipline of modeling } \\
\text { process, and ongoing critical reflections }\end{array}$ \\
$\begin{array}{ll}\text { Commitment to practitioner ownership and assessment } \\
\text { of the model }\end{array}$ & $\begin{array}{l}\text { Revising, redirecting, starting over as needed, until the model } \\
\text { "fits" }\end{array}$ \\
\hline
\end{tabular}


complexity of their work in the community. Beyond the first impressions, the visual presentation contains a remarkable amount of information and tends to be more accessible than lengthy written or spoken descriptions might be. Viewers tend to get drawn in, tracing significant pathways and noticing interesting outcomes, or examining parts of the work that they have had some hand in whether as a staff member, volunteer, program participant, or interested outsider. Large-format posters of the CPMs are on display in several of the community partner offices, providing ready background for discussions with board members, community collaborators, and others. O ne community partner used the structure of the model to create a template for internal quarterly reporting of activities and progress toward key outcomes. Several community partners have used the models in subsequent grant applications as a way of conveying not only the nature and strategy of their work, but also their clarity of thought and commitment to communication and accountability. Two community partners have incorporated CPM into new projects launched or proposed since the Food Dignity project concluded.

By bringing to the surface the strategic thinking and theory of change that shape the work on the ground of community-based programs, Collaborative Pathway Modeling provides a practical tool for bringing forward an essential part of the body of knowledge needed to ensure effective programmatic and policy responses to contemporary challenges. The need for additional sources of knowledge in general, and for innovative, community-designed approaches in particular, is underscored by problems and shortfalls that have been observed as evidencebased, research-derived programs have been implemented in real-world contexts (Horowitz, Robinson, \& Seifer, 2009; Lobb \& Colditz, 2013; Seifer \& Sisco, 2006; Wandersman et al., 2016). Pine and de Souza (2013) issued a particular call for including people with lived experience of food insecurity into "an expanded program of food scholarship" (p. 71). Collaborative Pathway Modeling offers an innovative, ethically grounded tool that can contribute to the way forward.

\section{Acknowledgments}

The authors are grateful to the staff and leadership of the community organizations partnering in the Food Dignity project who collaborated so deeply with us in the development of the Collaborative Pathway Models presented here, and in particular to G ayle Woodsum, whose insight shaped CPM development and who contributed valuable feedback on early drafts of this manuscript.

\section{References}

Ahmed, S. M., \& Palermo, A. S. (2010). Community engagement in research: Frameworks for education and peer review. A merican Journal of Public $\mathrm{H}$ ealth, 100(8), 1380-1387. https:// doi.org/ 10.2105/ ajph.2009.178137

Askew, K., Beverly, M. G., \& Jay, M. L. (2012). Aligning collaborative and culturally responsive evaluation approaches. E valuation and Program Planning, 35(4), 552-557. https:// doi.org/ 10.1016/ j.evalprogplan.2011.12.011

Chen, H. T., \& Turner, N. C. (2012). Formal theory versus stakeholder theory: New insights from a tobacco-focused prevention program evaluation. A meriman Journal of $\mathrm{E}$ valuation, 33(3), 395-413. https:// doi.org/ 10.1177/ 1098214012442802

Christopher, S., Watts, V., McCormick, A. K. H. G ., \& Y oung, S. (2008). Building and maintaining trust in a communitybased participatory research partnership. A merican Journal of Public $\mathrm{H}$ ealth, 98(8), 1398-1406. http:// doi.org/ 10.2105/ AJPH.2007.125757

Cochran, P. A., Marshall, C. A., Garcia-D owning, C., Kendall, E., Cook, D., McCubbin, L., \& Gover, R. M. S. (2008). Indigenous ways of knowing: Implications for participatory research and community. A merican Journal of Public H ealth, 98 (1), 22-27. https:/ / doi.org/ 10.2105/ ajph.2006.093641

Community-Campus Partnerships for Health. (n.d.). Annual award: 2014 CCPH Award Recipient: Food Dignity. Retrieved from https:// docs.wixstatic.com/ ugd/ c0c10a 83837b727dfb49a4a7b65cee67764d91.pdf

Corbie-Smith, G., Thomas, S. B., \& St. G eorge, D. M. M. (2002). Distrust, race, and research. A rchives of Internal M edicine, 162(21), 2458-2463. https:// doi.org/ 10.1001/ archinte.162.21.2458 
Journal of Agriculture, Food Systems, and Community Development

ISSN: 2152-0801 online

https:/ / www.foodsystemsjournal.org

Drahota, A., Meza, R. D., Brikho, B., Naaf, M., Estabillo, J. A., Gomez, E. D ., ... Aarons, G. A. (2016). Communityacademic partnerships: A systematic review of the literature and recommendations for future research. The Millbank Q uarterly, 94(1), 163-214. https:// doi.org/ 10.1111/ 1468-0009.12184

Evans, G. L. (2013). A novice researcher's first walk through the maze of G rounded Theory: Rationalization for Classical G rounded Theory. G rounded Theory Review, 12(1), 37-55. Retrieved from http:/ / groundedtheoryreview.com/ 2013/ 06/ 22/ a-novice-researchers-first-walk-through-the-maze-of-groundedtheory-rationalization-for-classical-grounded-theory/

Glaser, B. G., \& Strauss, A. L. (1965). D iscovery of substantive theory: A basic strategy underlying qualitative research. The A merican Behavioral Scientist, 8(6), 5-12. https:/ / doi.org/ 10.1177/ 000276426500800602

Green, L. W. (2001). From research to 'best practices' in other populations and settings. A merican Journal of $H$ ealth Behavior, 25(3), 165-178. https:/ / doi.org/ 10.5993/ ajhb.25.3.2

Green, L. W., \& Mercer, S. L., (2001) Can public health researchers and agencies reconcile the push from funding bodies and the pull from communities? A merican Journal of Public $\mathrm{H}$ ealth, 91(12), 1926- 1929. https:/ / doi.org/ 10.2105/ ajph.91.12.1926

Horowitz, C. R., Robinson, M., \& Seifer, S. (2009). Community-based participatory research from the margin to the mainstream: Are researchers prepared? Circulation, 119(19), 2633-2642. https:// doi.org/ 10.1161/ CIRCULATIONAHA.107.729863

Leviton, L. C., \& Trujillo, M. D . (2016) Interaction of theory and practice to assess external validity. E valuation Review 41(5), 1-36. https:// doi.org/ 10.1177/ 0193841X15625289

Lobb, R., \& Colditz, G . A. (2013). Implementation science and its application to population health. A nnual Review of Public H ealth, 34 , 235-251. https:/ / doi.org/ 10.1146/ annurev-publhealth-031912-114444

Minkler, M. (2005). Community-based research partnerships: Challenges and opportunities. Journal of U rban $\mathrm{H}$ ealth, 82(Suppl. 2), ii3-ii12. https:/ / doi.org/ 10.1093/ jurban/jti034

Pine, A. M., \& de Souza, R. (2013). Including the voices of communities in food insecurity research: An empowermentbased agenda for food scholarship. Journal of A griculture, Food Systems, and Community D evelopment, 3(4), 71- 79. https:// doi.org/ 10.5304/ jafscd.2013.034.007

Porter, C. M., Herrera, H., Marshall, D., \& Woodsum, G. M. (2014). Shared voices, different worlds: Process and product in the Food Dignity action research project. $\mathrm{G}$ ateways: International Journal of $\mathrm{C}$ ommunity Research and E ngagement, 7(1), 116-128. http:// dx.doi.org/ 10.5130/ ijcre.v7i1.3399

Seifer, D ., \& Sisco, S. (2006). Mining the challenges of CBPR for improvements in urban health. Journal of U rban $H$ ealth, 83(6), 981-984. https:/ / dx.doi.org/ 10.1007\%2Fs11524-006-9112-z

Trochim, W., Urban, J. B., Hargraves, M., Hebbard, C., Buckley, J., Archibald, T., ... Burgermaster, M. (2016). The guide to the Systems E valuation Protocol (V 3.1). Ithaca, New York: Cornell Digital Print Services. Retrieved from https:/ / core.human.cornell.edu/ research/ systems/ protocol/ indexlinks.cfm

Tseng, V. (2015, O ctober 27). Evidence at the Crossroads, Pt 1: What works, tiered evidence, and the future of evidence-based policy. [William T. G rant Foundation blog post]. New York: William T. G rant Foundation.Retrieved from http:// wtgrantfoundation.org/ evidence-at-the-crossroads-pt-1-what-works-tieredevidence-and-the-future-of-evidence-based-policy

Urban, J., \& Trochim, W. (2009). The role of evaluation in research-practice integration: Working toward the "Golden Spike." A merican Journal of E valuation, 30(4), 538-553. https:/ / doi.org/ 10.1177/ 1098214009348327

Wallerstein, N., \& D uran, B. (2010). Community-based participatory research contributions to intervention research: The intersection of science and practice to improve health equity. A merican Journal of Public H ealth, 100(S1), S40-S46. https:/ / doi.org/ 10.2105/ ajph.2009.184036

Wandersman, A., Alia, K., Cook, B. S., Hsu, L. L., \& Ramaswamy, R. (2016). Evidence-based interventions are necessary but not sufficient for achieving outcomes in each setting in a complex world: Empowerment evaluation, getting to outcomes, and demonstrating accountability. American Journal of E valuation, 37(4), 544-561. https:/ / doi.org/ 10.1177/ 1098214016660613 


\section{Appendix. Summary of Steps and Guidance for Collaborative Pathway Modeling (CPM)}

\begin{tabular}{|c|c|}
\hline CPM Steps & Key Considerations and Guidance for CPM Process \\
\hline Begin the collaboration & $\begin{array}{l}\text { - Hold these points firmly in mind: } \\
\text { - Humility (the goal is to surface what, by definition, you do not know) } \\
\circ \text { Trust is essential } \\
\text { The goal is to bring to the surface and articulate, in formal modeling } \\
\text { structures, the strategic thinking, key assumptions, operating principles, } \\
\text { insights about context, and theory of change held by the community } \\
\text { organizers and colleagues } \\
\text { - Ensure shared understanding of purpose, audience, relevant contributors, } \\
\text { timeline, etc. } \\
\text { - Establish clearly that decision-making control, final approval, and ownership } \\
\text { of the resulting model are held by the community organization. }\end{array}$ \\
\hline Advance preparation & $\begin{array}{l}\text { - With community organizer help, identify all available materials describing } \\
\text { the project or program (website, internal documents, grant proposals, media } \\
\text { coverage, etc.). } \\
\text { - Review and make notes of all content that could be considered activities, } \\
\text { major or intermediate outcomes, broad components of the work, or relevant } \\
\text { contextual information. } \\
\text { - Create a rough sketch or pre-model to organize this material to the extent it } \\
\text { is possible, identifying questions and issues to be clarified. } \\
\text { - If useful, enter pre-model into the web-based Netway software program } \\
\text { (https:// www.evaluationnetway.com). }\end{array}$ \\
\hline Site visit: Planning for it & $\begin{array}{l}\text { - Talk with community organizer to determine who will be the important } \\
\text { contributors to the model-individuals with diverse perspectives or roles in } \\
\text { the work can be very valuable, but it is also appropriate to build a model of a } \\
\text { single individual's vision. } \\
\text { - Arrange site visit, scheduling sizeable blocks of time for individual or group } \\
\text { interviews, as well as time for a closing presentation to present and get } \\
\text { feedback on the draft model. }\end{array}$ \\
\hline Site visit: Gathering input & $\begin{array}{l}\text { - Invite contributors to talk about the work and help you to understand what } \\
\text { they do and why. Adapt prompt questions to the conversation, with the goal } \\
\text { of filling out your understanding of the scope and workings of the program. } \\
\text { - Listen carefully, try to hear and learn as much as possible about what they } \\
\text { (or the organization) does, why they do it, what changes they've seen, what } \\
\text { changes they anticipate and why, what changes are difficult to achieve and } \\
\text { what happens relatively easily, why the changes matter, who participates in } \\
\text { the program, who is affected by it, what differences it makes, etc. } \\
\text { - Pay attention to what is unfamiliar, or to what you may be making } \\
\text { assumptions about. Explore these, to ensure that their insights and } \\
\text { reasoning are what you work with. } \\
\text { - Listen with an ear attuned to the eventual goal of developing a model with } \\
\text { clear causal story lines that connect elements of the work to the changes } \\
\text { they engender and ultimately to the big-picture goals. } \\
\text { - Take extensive notes. }\end{array}$ \\
\hline $\begin{array}{l}\text { Site visit: Synthesizing, } \\
\text { distilling, and building the } \\
\text { model }\end{array}$ & $\begin{array}{l}\text { - Review notes and highlight all the elements that could have a place in the } \\
\text { model and need to be incorporated in some way. } \\
\text { - Reflect on the appropriate scope-what are the boundaries for current } \\
\text { modeling purposes? What parts of the information need to be in the active }\end{array}$ \\
\hline
\end{tabular}


working model; what parts are important as explanatory background or context, or important assumptions underlying the work?

- Reflect on the level of detail or generality needed for the scope of work being modeled-smaller projects can be modeled with more specificity, larger-scope projects will need to use broader characterizations (for example, by bundling several small specific activities under a broader title that includes and characterizes them all); similarly, individual specific outcomes might need to be bundled into broader constructs. Often, the right level of detail will not be clear until the model is more developed, but be prepared for the choices that will need to be made. (There is no definitive rule about the correct level of detail. Key considerations are that the final model be readable, and that there be enough detail to provide meaningful insights and meet the preferences and needs of the community organizer.)

- Transfer the emerging individual elements to index cards, and lay them out on a large surface in the ways that contributors connected them in their explanations and descriptions.

- Review, reflect, adjust, edit, add and delete or combine elements, return to notes and make sure you haven't missed anything, return to the cards and see if the threads of the story are there and connected in the ways they should be.

- Enter the rough draft into the Netway, see how it looks, print it out and again review, reflect, adjust, edit, etc.

- Get the model to the place where it "hangs together" and tells what you understand to be the story.

Site visit: Present the draft model to the assembled contributors, get feedback
- Bring printouts of the draft model (large enough for people to read).

- Offer an introduction to the model, pointing out the main long-term goals, major activities, "regions" that show up in the model reflecting the major threads of the work, significant outcomes along the way, and so on as appropriate.

- Give them time to review the model on their own, and to ask questions of the CPM team.

- Invite their feedback on what looks right, what looks wrong, what's missing, what they like or don't like, anything that surprises them, etc.

- Discuss and agree on changes to be made.

Finalize model

- Make revisions to the model in the Netway, send or show the revised model to the community organizer and key contributors, invite further improvements and corrections.

- Continue until they feel that the model matches their understanding of their work and its design and purposes, and they give final approval.

- Prepare final print and digital versions of the model, including citation information and appropriate credits. 